\title{
REVIEW
}

\section{Hypersensitivity to Orthopedic Implants: A Review of the Literature}

\author{
Joseph Wawrzynski · Joseph A. Gil · Avi D. Goodman · Gregory R. Waryasz
}

Received: October 14, 2016/Published online: March 31, 2017

(C) The Author(s) 2017. This article is an open access publication

\begin{abstract}
Awareness of rare etiologies for implant failure is becoming increasingly important. In addition to the overall increase in joint arthroplasties, revision surgeries are projected to increase dramatically in the coming years, with volume increasing up to seven-fold between 2005 and 2030. The literature regarding the relationship between metal allergy and implant failure is controversial. It has proven difficult to determine whether sensitization is a cause or a consequence of implant failure. Testing patients with functional implants is not a clinically useful approach, as the rate of hypersensitivity is higher in implant recipients than in the general population, regardless of the status of the implant. As a result of the ineffectiveness of preoperative patch testing for predicting adverse outcomes, as well as the high cost of implementing such patch testing as standard
\end{abstract}

Enhanced content To view enhanced content for this article go to http://www.medengine.com/Redeem/ 1508F06019AC8C24.

J. Wawrzynski

Warren Alpert Medical School, Brown University, Providence, RI, USA

J. A. Gil ( $\varangle)$ · A. D. Goodman · G. R. Waryasz Department of Orthopaedic Surgery, Warren Alpert Medical School, Brown University, Providence, RI, USA

e-mail: joseph_gil@brown.edu procedure, most orthopedists and dermatologists agree that an alternative prosthesis should only be considered for patients with a history of allergy to a metal in the standard implant. In patients with a failed implant requiring revision surgery, hypersensitivity to an implant component should be considered in the differential diagnosis. Because a metal allergy to implant components is currently not commonly considered in the differential for joint failure in the orthopedic literature, there should be improved communication and collaboration between orthopedists and dermatologists when evaluating joint replacement patients with a presentation suggestive of allergy.

Keywords: Contact

dermatitis; Hypersensitivity; Metal implants; Orthopedic implants; Patch testing

\section{INTRODUCTION}

Hypersensitivity to implanted metal orthopedic devices, and the relevant sequelae, has been the source of much debate in the literature. While reports of cutaneous reactions following skin exposure to items containing metal are relatively common, similar reactions to implanted metallic orthopedic devices are considerably rarer. The first report of cutaneous hypersensitivity caused by a metallic orthopedic implant 
was a 1966 case report by Foussereau and Laugier [1], which described a patient with eczematous dermatitis overlying the site of a metallic plate used for fracture fixation [2]. Since then, many case reports have described similar cutaneous reactions to metallic implants, including cardiovascular, dental, plastic surgical, and orthopedic varieties. Other potential manifestations of metal hypersensitivity from implants include impaired wound healing, infection-mimicking reactions, effusions, pain, and implant loosening [3].

Patients often report hypersensitivity reactions to metals found in costume jewelry, belt buckles, or watches, and hypersensitivity reactions to metals manifesting as contact dermatitis are quite common in the general population [4]. The most common metal sensitizer in humans is nickel, although beryllium, cobalt, chromium, tantalum, titanium, and vanadium have also been shown to induce hypersensitivity responses in tissues. The overall prevalence of metal hypersensitivity in the general population is estimated to be between $10 \%$ and $15 \%$, higher in women than in men $[5,6]$. Contact allergy to nickel, cobalt, and chromium is seen in $15.5 \%, 6.2 \%$, and $2.3 \%$, respectively, of the North American population [7]. However, the prevalence of metal allergy appears to be influenced by age. In a study of 493 trauma patients with a mean age of 39.24 , Swiontkowski et al. found the prevalence of sensitivity to chromium, nickel, and cobalt to be $0.2 \%, 1.3 \%$, and $1.8 \%$, respectively, considerably lower than the rates reported in the general population, leading the authors to suggest that metal sensitivity may be less prevalent in the younger population [8].

The diagnostic tool most commonly used to confirm or rule out a metal allergy is the patch test. The metals tested as part of the North American Contact Dermatitis Group (NACDG) standard series for patch testing includes nickel, chromium, cobalt, and gold; however, other metals such as titanium, vanadium, palladium, tin, copper, aluminum, mercury, and silver may also be tested, depending on the patient's clinical history [9]. Patch test preparations also often include components of the implanted device and cement such as gentamicin sulfate, benzoyl peroxide, hydroquinone, 2-hydroxyethylmethacrylate, copper(II) sulfate, methylmethacrylate, $N, N$-dimethyl- $p$-toluidine [3].

Metal hypersensitivity often manifests as contact dermatitis on the area of skin that was exposed to the metal; however, metallic orthopedic implants are inserted deep within the tissue and away from the skin. Nevertheless, these metals can sensitize the body and provoke an immune reaction. According to Aquino and Mucci, "systemic contact dermatitis (SCD) refers to a condition where an individual who is sensitized to an allergen via the cutaneous route will subsequently react to that same allergen or a cross-reacting allergen via the systemic route (oral, intravenous, intramuscular, inhalational, transmucosal, or transcutaneous)". This secondary reaction can manifest as a skin reaction such as dermatitis, urticaria, bullous reactions, vasculitis, or impaired wound healing at the site of the initial reaction or elsewhere, or it may be related to the type IV delayed hypersensitivity reaction sometimes observed in the periprosthetic tissues of metallic implant recipients $[10,11]$.

Much of the literature surrounding metal implant hypersensitivity has focused on the correlation between the hypersensitivity response and implant failure. Circulating metal ions increase following joint replacement, especially in patients who have experienced implant loosening. This increase has been attributed to the accumulation and continual release of metal ions into the tissue surrounding the implant as a result of the inevitable corrosive process. These metal ions may trigger a localized inflammatory response that can lead to implant failure $[5,12]$. This proposed etiology is controversial, however. Metal hypersensitivity in patients with a failed implant is approximately six times more common than in the general population, and three times higher than in all patients with a metallic implant [5]. It is not known, however, whether this hypersensitivity is the cause or the result of implant failure, or whether separate autoimmune mechanisms may be responsible. It also remains a subject of debate whether metal hypersensitivity should impact treatment decisions in orthopedic implant candidates $[4,5,13,14]$. 
This article is based on previously conducted studies and does not involve any new studies of human or animal subjects performed by any of the authors.

\section{ETIOLOGY}

The hypersensitivity reaction is typically in an immune response mounted against metallic particles that are released as a result of implant wear or corrosion. This was particularly an issue with metal-on-metal hip joint replacements, which were initially popularized for their durability, lower rate of hip dislocation, bone conservation, and superior joint mechanics [15]. Loose metallic degradation products, also known as haptens, can complex with proteins and become capable of acting as antigens for circulating lymphocytes. In doing so, haptens induce an immediate humoral hypersensitivity response characterized by the formation of antibodies and immune complexes (type I, II, and III hypersensitivity reactions), but more commonly they induce a cell-mediated delayed-type (type IV) hypersensitivity response via the activation of $\mathrm{CD} 4+\mathrm{T}_{\mathrm{H}} 1$ lymphocytes in the peripheral lymphoid tissues [5]. These $\mathrm{T}_{\mathrm{H}} 1$ lymphocytes release pro-inflammatory cytokines (i.e., TNF- $\alpha$, IFN- $\gamma$, IL-1, and IL-2), which recruit macrophages to the site of the implant [16].

In their study of 19 metal-on-metal hip arthroplasty revisions, Willert et al. noted that the additional presence of B lymphocytes, plasma cells, and massive fibrin exudation at the site of the revision distinguished the reaction from a typical type IV hypersensitivity reaction, and instead referred to the reaction as an aseptic lymphocytic vasculitis-associated lesion (ALVAL) [17]. Nonetheless, in these cases the authors state that the histopathology is consistent with a cell-mediated immune response. This association between hypersensitivity to orthopedic implants and cell-mediated immunity has been supported by the discovery of T lymphocytic infiltrate at the site of removed prostheses, as well as the resolution of hypersensitivity upon the removal of the implant or administration of immunosuppressive medication [12]. Significant levels of metal ions have been found in the periprosthetic tissues, as well as the liver, spleen, lymph nodes, and urine/serum of hip arthroplasty patients, demonstrating that this is more than a purely localized phenomenon [18]. Vermes et al. measured the in vitro proliferation of peripheral human leukocytes to metallic antigens prior to and at regular intervals following hip replacement surgery, and found that hypersensitivity had developed in $18 \%$ of patients following cementless hip replacement by 36 months after surgery. The authors note that the percentage of the cohort with metal sensitivity was dependent on the length of exposure time to the implant, with the number increasing from $12 \%$ to $18 \%$ from 6 to 36 months after surgery [16].

Alternatively, hypersensitivity to metallic implants may be a reaction to separate components of the implant, including allergens in the bone cement such as acrylates, benzoyl peroxide, $N, N$-dimethyl- $p$-toluidine, and gentamicin. Bircher et al. reported on five patients experiencing complications following knee or shoulder joint replacement, who were subsequently found to be contact allergic to benzoyl peroxide. Reactions in these patients included pain, swelling, pruritis, and implant loosening [19]. A rare manifestation of hypersensitivity to an implant component was reported by Maldonado-Naranjo et al.: an allergic reaction characterized by itching, erythema, periorbital swelling, and macroglossia due to the polyetheretherketone (PEEK) in an intervertebral cage used in spinal surgery [20].

A potential etiology for aseptic joint loosening involves the proliferation of osteoclasts on metals such as titanium and vanadium and their subsequent degradation, uptake, and release of the metals from the device. This would account for the high serum levels of metal ion that are often found in systemic circulation, and would serve as a potential mechanism for implant loosening. This degradation process may also induce a hypersensitivity reaction that exacerbates the loosening process [18]. A separate proposed mechanism involves the haptenogenic stimulation of toll-like receptors in the periprosthetic tissue. Nickel, for example, has been shown to stimulate TLR4 
[11]. Still others suggest that the mechanism for implant loosening is primarily the result of a cytotoxic process in response to prolonged wear on the joint. This toxic etiology is supported by the fact that retrieval studies have found that most failures of components are the result of increased wear. These authors concede that implant failures in patients with normal wear may result from hypersensitivity [15].

\section{CLINICAL MANIFESTATIONS}

While metal sensitivity contributing to implant failure may be obvious, its manifestations may also be subtle. Implant-related hypersensitivity is very difficult to diagnose, so its true prevalence is likely underreported [5]. Implant sensitivity presenting as a cutaneous reaction is rare, and Kubba et al. tallied only a total of 19 such patients out of a total of 800 with this presentation over a 2 -year prospective study performed at the Cleveland Clinic. The authors suggest that more subtle or transient skin reactions likely would not have prompted a return trip to the hospital among affected patients [21]. Krecisz et al. [22] studied contact allergies to metals in 14 metallic implant recipients (nine women and five men) with symptoms including recurrent skin eruptions (either generalized or nearby the implants), and the formation of sterile fistulas in three patients. They found that eight of 14 patients demonstrated allergic symptoms to metals present in the prostheses within 1 year of operation. Three of the eight allergic patients had been re-operated on using non-allergic prostheses by the time of publication and their symptoms disappeared.

There is also evidence that metal sensitivity may play a role in causing the conditions of chronic fatigue syndrome (CFS), fibromyalgia, and autoimmune/inflammatory syndrome induced by adjuvants (ASIA). Stejskal reported the cases of five patients presenting with CFS or fibromyalgia symptoms, each with histories of metal implantation. Lymphocyte transformation testing (LTT) was positive for a metal contained in the implant in each case. The author noted that an increased prevalence of allergy to foods and other common allergens in CFS patients has been reported previously [23].

Conflicting data exists regarding the impact of metal hypersensitivity on arthroplasty failure. On the basis of the findings of their review, Hallab et al. state that there is currently no evidence that patch test reactivity increases a patient's risk for an allergic reaction to a prosthesis; however, an increased risk does exist in patients with a history of an allergic reaction to a metallic device or jewelry [5]. Granchi et al. studied metal hypersensitivity in 94 total knee arthroplasty (TKA) patients via patch test and found that while patch testing was unable to differentiate between stable and failed implants, implant failure was four times as likely if the patient had symptoms of metal sensitivity before the surgery [24].

In contrast, some studies have found that patients with a preexisting cutaneous hypersensitivity to metal became desensitized following implantation of a metallic orthopedic implant. In a prospective study performed by Rooker and Wilkinson in which six patients who tested positive for metal hypersensitivity via patch test prior to metal implantation, five had lost their sensitivity upon retesting at 3-19 months post-operatively [25]. This phenomenon was also observed by Carlsson and Möller [26], who found that three of 18 patients involved in their 1988 study had been desensitized to the metal antigen upon follow-up patch testing [5]. These authors acknowledged that their results might, in fact, have been reflective of the unreliability of the patch testing method. Aquino and Mucci present a retrospective study involving 356 hip arthroplasty patients, which found no difference in revision rates between allergic and non-allergic patients. The authors also present a prospective study involving 18 patch test-reactive patients that found that although no complications emerged in any of these patients, the life of the implant was shorter on average [11].

\section{DIAGNOSIS}

When evaluating a joint replacement patient with suspected immune-related prosthetic 
failure, it is important to first rule out intra-articular infection. The non-allergic causes of implant failure include aseptic osteolysis, infection, recurrent dislocation, and fracture [10]. Gawkrodger notes that although culturing tissue samples and swabs from the affected area is often considered the gold standard for diagnosis, false positives and false negatives are common. The author states that consequently histopathology has been more widely adopted as the most accurate test. An arthroscopic biopsy can be performed if hypersensitivity is suspected, in which case an intraoperative frozen section of the periprosthetic membrane is ideal for diagnosis [27]. According to Galbraith et al., the histological appearance of metal-on-metal joint failures consists of "a diffuse collection of perivascular lymphocytes, plasma cells, localized bleeding, necrosis, fibrin exudation, and presence of macrophages with drop-like inclusions" [28]. In distinction, infection of the periprosthetic tissue is marked by an abundance of neutrophils. Although it has been shown to be ineffective at differentiating between a stable and a failed implant, patch testing has been found to be useful in predicting the survival curve of a patient's implant. In a study conducted by Granchi et al. involving 223 total hip replacement recipients and candidates, the median duration of the implant was 78 months in patients with positive patch test. This was significantly less $(p=0.04)$ than the median of 120 months found in patients who exhibited no skin reaction [29].

Although hypersensitivity may play a role in implant failure, a distinction exists between the cutaneous immune reaction to metals, as manifested by patch testing, and the periprosthetic hypersensitivity to metallic implants. Patch test reactivity has been suggested as a potential method to screen for patients at risk of developing a hypersensitivity reaction to a metallic implant. Recent guidance from the German Contact Allergy Group (DKG), however, stresses that patch testing should only be used as a tool to "verify or exclude metal allergy in patients with a corresponding history". The group advises against prophylactic screening of implant candidates using patch testing [30].
Schalock and Thyssen surveyed 119 dermatologists at the 2012 European Society of Contact Dermatitis and 2013 American Contact Dermatitis Society meetings and came up with four major and five minor diagnostic criteria for contact dermatitis secondary to metallic implant. The designated major criteria (each identified by approximately $80 \%$ of dermatologists) include eruption overlying the metal implant, positive patch test reaction to a metal used in the implant, complete recovery after removal of the offending implant, and chronic dermatitis beginning weeks to months after metallic implantation. The characteristics identified as minor criteria (which were selected by $60 \%$ or fewer of the surveyed dermatologists) include dermatitis reaction is therapy resistant, morphology consistent with dermatitis (erythema, induration, papules, vesicles), systemic allergic dermatitis reaction, histology consistent with allergic contact dermatitis, and positive in vitro test to metals (e.g., the lymphocyte transformation test) [31].

In patients presenting with a suspected allergic reaction to a metallic implant in the absence of implant failure, Thyssen et al. suggest that the workup should include patch testing and consideration of in vitro peripheral lymphocyte proliferation testing (lymphocyte transformation test, or LTT). The test compares peripheral blood lymphocyte proliferation upon 7-day incubation with and without the addition of metal antigen. LTT is a helpful test in doubtful or questionable cases. The authors note that $2+$ and $3+$ patch test reactions are more consistent with complications related to the metal implant than milder reactions [10]. LTT more directly tests the immune components responsible for the hypersensitivity reaction in the periprosthetic tissue than the patch test, as the antigen-presenting cell in the skin (the Langerhans cell) is not present in the deeper tissue. Müller and Valentine-Thon performed a study involving 56 individuals with $\mathrm{Ti}$ implants, systemic symptoms, and negative patch test results. A Ti LTT was performed on each of these patients and came back positive in 21 cases $(37.5 \%)$, despite the negative patch test results $[18,32]$. Despite these promising results, the LTT has yet to earn widespread adoption 
among providers evaluating cases of suspected metal allergy [31]. The authors found that 89\% of those surveyed always use patch testing, while $83 \%$ never use LTT to evaluate metal dermatitis. They cite logistic complications including access to laboratories, price, and the inability to test for certain metals as reasons for the lack of wider LTT utilization [7].

In a questionnaire conducted by Schalock and Thyssen of attendees of the 2013 European Society of Contact Dermatitis and 2013 American Contact Dermatitis Society meetings, the authors found that $54 \%$ of 119 respondents believed that patients with suspected moderate to severe metal dermatitis should be patch tested and/or offered LTT prior to metal implantation, while $38 \%$ believed that simply counseling a surgeon to use a less immunogenic titanium device was sufficient. Of note, only $8 \%$ of respondents felt that no testing or change in management was necessary [7]. In 2016, Schalock et al. reported guidelines regarding patch testing based on existing evidence and expert opinion [33]. Routine preoperative patch testing of patients with no history of contact dermatitis to metal is not indicated. However, patients who have a clear history of contract dermatitis to metal should be evaluated by patch testing prior to device implantation. In patients who are suspected to have contact dermatitis secondary to an implanted device, a positive patch test alone should not be used to justify explantation of the device. The decision to explant a device should be made on the basis of an assessment of all clinical factors and a risk-benefit analysis done by the patient and physician. Atanaskova Mesinkovska et al. suggest that all patients with a history of hypersensitivity to metals should undergo preoperative patch testing. The authors state that this practice allows for other potential sensitizers to be identified, adding that $74 \%$ of patients receiving preoperative patch test due to self-reported metal hypersensitivity were reactive to more than one of the metals tested. Of 31 patients in the study who identified the metal hypersensitivity preoperatively, 21 received an allergen-free implant and had outcomes free of hypersensitivity. In 6 of 10 patients who recognized a metal allergy after implantation, the metallic device was removed and symptoms resolved. The authors believe that awareness of these concurrent allergies preoperatively allows surgeons to more effectively select an appropriate metal prosthesis in each case, minimizing the potential for complications [4]. Complicating this approach, a review of the literature conducted by Rostoker et al. found that of 42 patients who experienced orthopedic implant dermatitis due to an immunological mechanism, only $29 \%$ had a prior history of metal contact dermatitis [34]. Razak et al. conducted a survey of orthopedic surgeons in the UK, evaluating their opinions on the utility of preoperative patch test screening for metal allergy in implant candidates, and the consensus opinions among the 18 surgeons who responded were that preoperative patch testing was not necessary and that cobalt-chromium or stainless steel implants should be used in patients with a suspected metal allergy regardless of the results of patch testing [35].

Although the diagnosis of metal allergy in an implant candidate can be challenging and uncertain, the trend among clinicians is to offer patch testing to patients with a history of metal allergy and to choose a less immunogenic device for implantation. In patients presenting with dermatitis after implantation of a metal device, arthroscopic biopsy and histological analysis of the periprosthetic tissue or lymphocyte transformation testing appear to be more accurate tests for diagnosing metal hypersensitivity than patch testing.

\section{IMPACT ON OUTCOMES}

The impact of preexisting metal sensitivity on outcomes, as obtained from preoperative history or patch testing, remains controversial. Granchi et al. found that the median life span of a hip prosthesis decreased from 120 to 78 months in patients with a positive patch test [29]. In their prospective clinical study, Frigerio et al. found that patient history was far less reliable than patch testing for discovering metal sensitivity. As a result, the authors recommend that patch testing and LTT should be performed on patients during the planning stages of a total 
joint replacement [36]. Recently, however, Bravo et al. designed a retrospective matched control study involving 161 TKA procedures performed on 127 patients over 12 years to evaluate the efficacy of a positive preoperative skin patch test as a predictor of future implant-related complications, adverse clinical outcomes, and decreased prosthetic survivorship. Patch test-positive patients were those who tested positive to cobalt-chromium, stainless steel, and titanium-aluminum-vanadium, the most commonly used alloys in orthopedic prostheses. The authors found no significant difference in complications, reoperation, revisions, and functional outcomes between patch test-positive patients, patch test-negative patients, and matched controls, noting that the revision rate at 5 years was $1.9 \%$ for patch test-positive patients (vs. $2.4 \%$ for matched controls), and $0 \%$ for patch test-negative patients (vs. 15\% for matched controls). The authors suggest that no causal relationship can be made between patch test reactivity and implant performance [37].

In 2012 Granchi et al. published a systematic review and meta-analysis of the literature pertaining to metal sensitivity testing in joint replacement patients and similarly concluded that preoperative hypersensitivity testing was unable to discriminate between stable and failed total joint replacements. The authors state that they were only able to find one prospective cohort study evaluating metal hypersensitivity in stable versus failed joint replacements, and the study found that the patch test offered no predictive value as it was never positive in those with failed joint replacements [12]. So et al. studied the outcomes in ankle fractures fixed with metallic implants, with 60 men and 46 women undergoing treatment. The authors found that neither a history of metal hypersensitivity nor positive preoperative patch test correlated with poor outcomes in these patients, including implant revision rates and self-reported pain [38].

\section{TREATMENT}

One of the early case reports implicating metal hypersensitivity in orthopedic implant failure involved a 22-year-old woman with stainless steel screws implanted in her patella 5 months prior, who presented with eczematous dermatitis on her chest and back. After several unsuccessful attempts at treatment, the screws were eventually removed, and the cutaneous reaction subsided completely within $72 \mathrm{~h}$ [5].

In cases of hypersensitivity-mediated reactions where a metal implant is implicated as the cause, replacement of the prosthesis is typically the most effective treatment $[2,20,39-42]$. As a result of the invasive nature and associated complications of this approach, however, other treatment options can be considered first. A 21-day course of topical corticosteroids at the site of a cutaneous reaction has been shown to be effective in some patients [2]. In patients where such a treatment course is ineffective or unrealistic, treatment options include removing an implant that has served its purpose, replacing an implant with a non-allergic alloy, and using an implanted device that has been coated with polytetrafluoroethylene [5, 11]. Basko-Plluska et al. concur with this guidance, adding that replacement should not be considered in patients with a perfectly functioning implant causing no pain with a positive patch test result [43]. Mowad suggests that patch testing may be most beneficial to assess cases of dermatitis overlying a static implant, because such patients who are patch test positive tend to respond best to implant removal [39]. Surgical removal of the culprit metal in these cases results in a clearing of the hypersensitivity reaction. Thyssen et al. describe the case of a 12-year-old child who developed systemic allergic dermatitis secondary to the implantation of a nickel and cobalt plate in the patient's fractured leg 3 months prior. The dermatitis cleared rapidly upon removal of the implant. In the five fibromyalgia and CFS patients presented by Stejskal, all symptoms resolved following removal of the metal implant [23].

In 1987, Rostoker et al. [34] described the most commonly practiced prophylaxis for preventing orthopedic implant hypersensitivity as using titanium alloy. More recently, in their 2015 review article pertaining to shoulder arthroplasty in metal-sensitive patients, Morwood and Garrigues advocate for the use of 
titanium implants in preference to nickel varieties in patients with a history of cutaneous metal allergy owing to their comparable cost and lower likelihood of sensitization [44]. Although the majority of prostheses use cobalt-chromium alloys with a nickel content of less than $1 \%$, with a very low theoretical risk of infection, a case of a TKA patient who developed painful systemic allergic contact dermatitis has been reported; this very rare reaction was attributed to the chromium in the implant [2].

An innovation aimed at reducing the development of metal sensitivity to orthopedic implants is the development of coated implants. Lützner et al. conducted a randomized control trial of 120 TKA patients who received either a coated or an uncoated implant. Upon 1-year follow-up, the authors noted that serum metal ion concentrations had not increased in either group, sensitization was rare, and no skin reactions were observed. The authors state that longer-term follow-up will be necessary to further evaluate the impact of the coated implant [45].

In cases of implant failure where infection can be ruled out as a cause, Galbraith et al. recommend that a one-stage revision be performed. This reduces the length of hospital stay resulting from the need for surgical debridement of the joint and prolonged antibiotic administration. Replacement of the metal liner with an alternative bearing surface, or using a prosthesis made from a different metal alloy, should be considered $[10,28]$. The chromium-sensitive arthroplasty patient mentioned above underwent revision surgery using a zirconium-niobium alloy prosthesis, and the patient's dermatitis resolved without recurrence within 1 year [2].

In a case control study, Grammatopoulos et al. compared patients who had revision hip surgery due to metal hypersensitivity to those who had revision hip surgery due to fractures or other reasons, which included loosening, infection, avascular necrosis, and recurrent dislocations. The study found that outcomes, as measured by the Oxford hip score and the rate of complications, were much worse for the hypersensitivity group [46]. Citing the results of this study, Fary et al. suggest that the comparatively poorer outcomes among hypersensitivity patients may be related to the amount of existing tissue destruction at the time of surgery, and stress the importance of early identification of hypersensitivity-related implant failure in order to limit the extent of soft tissue destruction and osteolysis and improve the outcomes of revision surgery [15].

In patients diagnosed with hypersensitivity to metallic implant components, the most effective treatment is early replacement of the existing device with a device composed of a non-allergenic alloy. Use of implants with polytetrafluoroethylene-coated bearing surfaces is currently being investigated as an alternative approach. In patients where replacement of a device is unrealistic or a less invasive approach is preferable, a 21-day course of topical corticosteroids has been shown to be effective in some patients.

\section{METAL-ON-METAL JOINT PROSTHESIS}

Bolognesi and Ledford published a thorough history including current recommendations on the treatment of patients with metal-on-metal (MoM) hip prostheses [47]. The first MoM prosthesis was implanted in 1966 and it quickly gained popularity due to a theoretically lower wear-induced failure rate and the ability to produce a thinner acetabular component and correspondingly larger and more stable femoral head component relative to conventional metal-on-polyethylene prostheses. However, recent national registry data has demonstrated two to three times higher revision rates among MoM implant recipients than those receiving other types of implants. This has been attributed to the corrosion and release of metal particles and ions into the joint space, which instigates an immune reaction characterized by necrosis, osteolysis, large, sterile hip effusions, and pseudotumors, a constellation of traits that is now classified as an adverse reaction to metal debris (ARMD). In these cases, aseptic lymphocytic vasculitis-associated lesion (ALVAL) is the characteristic histopathological finding. The 
authors note that the US FDA publicly issued concerns regarding MoM hip prostheses in 2011, and three implant companies subsequently recalled their MoM prostheses because of high revision rates. The ideal management of patients with existing MoM prostheses is not yet well established. In asymptomatic patients with a MoM prosthesis, the authors recommend measuring metal ion levels in the blood, and monitoring the levels of patients with high ion concentrations ( $\geq 7 \mathrm{ppb}$ ) at 6- to 12-month intervals. However, the FDA has not yet determined the utility of regular monitoring of ion concentrations in asymptomatic implant recipients. Advanced imaging such as specialized metal artifact reduction sequence (MARS) MRI is recommended for either symptomatic implant recipients or asymptomatic recipients with elevated ( $\geq 7 \mathrm{ppb}$ ) metal ion concentrations. Ultrasonography is also gaining popularity as a diagnostic tool for evaluating fluid build-up in the periprosthetic tissues. The authors defer to the classification scheme developed by Fabi et al. [48] for the consideration of surgical treatment options in the event that revision surgery is necessary. In cases of suspected metal hypersensitivity, revision using a metal-on-polyethylene or ceramic-on-polyethylene bearing surface is recommended.

\section{CONCLUSIONS}

The literature regarding the relationship between metal allergy and implant failure is controversial. In one study of metal sensitivity in implant recipients, the rate of metal sensitivity was significantly higher among implant recipients than those without metallic implants $(p=0.005)$, but not significantly different between patients with stable and failed implants. The rates of metal sensitivity were $20 \%$ in the control group without an implant versus $59.6 \%$ in the group with an unstable metallic implant and $48.1 \%$ of patients with a stable implant $[6,24]$. As a result of this and other studies in which metal sensitivity was evaluated using patch tests or LTT and compared between stable and failed implant groups, it has proven difficult to determine whether sensitization is a cause or a consequence of implant failure [42]. Testing patients with functional implants is not a clinically useful approach, as the rate of hypersensitivity is higher in implant recipients than in the general population, regardless of the status of the implant [24].

As a result of the ineffectiveness of preoperative patch testing for predicting adverse outcomes, as well as the high cost of implementing such patch testing as standard procedure, most orthopedists and dermatologists agree that an alternative prosthesis should only be considered for patients with a history of allergy to a metal in the standard implant. In patients with a failed implant requiring revision surgery, hypersensitivity to an implant component should be considered in the differential diagnosis. Because a metal allergy to implant components is currently not commonly considered in the differential for joint failure in the orthopedic literature, there should be improved communication and collaboration between orthopedists and dermatologists when evaluating joint replacement patients with a presentation suggestive of allergy [42]. In the cases presented in the literature where metal hypersensitivity was diagnosed (via patch test or LTT) in a patient with a cutaneous reaction or implant failure, replacement of the implant with a non-allergic prosthesis resulted in the elimination of symptoms.

Awareness of rare etiologies for implant failure is becoming increasingly important. In addition to the overall increase in joint arthroplasties, revision surgeries are projected to increase dramatically in the coming years, with volume increasing up to seven-fold between 2005 and 2030 [42]. Though rare, it is important to understand metal hypersensitivity as a potential etiology for implant failure in order to select the proper replacement prosthesis and ensure a satisfying outcome whenever a revision is necessary.

\section{ACKNOWLEDGEMENTS}

No funding or sponsorship was received for this study or publication of this article. All named 
authors meet the International Committee of Medical Journal Editors (ICMJE) criteria for authorship for this manuscript, take responsibility for the integrity of the work as a whole, and have given final approval for the version to be published.

Disclosures. Joseph Wawrzynski, Joseph A Gil, Avi D. Goodman, and Gregory R. Waryasz have nothing to disclose.

Compliance with Ethics Guidelines. This article is based on previously conducted studies and does not involve any new studies of human or animal subjects performed by any of the authors.

Open Access. This article is distributed under the terms of the Creative Commons AttributionNonCommercial 4.0 International License (http://creativecommons.org/licenses/by-nc/4. $0 /$ ), which permits any noncommercial use, distribution, and reproduction in any medium, provided you give appropriate credit to the original author(s) and the source, provide a link to the Creative Commons license, and indicate if changes were made.

\section{REFERENCES}

1. Foussereau J, Laugier P. Allergic eczemas from metallic foreign bodies. Trans St Johns Hosp Dermatol Soc. 1966;52:220-5.

2. Gao X, He R, Yan S, Wu L. Dermatitis associated with chromium following total knee arthroplasty. J Arthroplasty. 2011;26:665.e13-6.

3. Thomas P. Clinical and diagnostic challenges of metal implant allergy using the example of orthopaedic surgical implants: Part 15 of the Series Molecular Allergology. Allergo J Int. 2014;23:179-85.

4. Atanaskova Mesinkovska N, Tellez A, Molina L, et al. The effect of patch testing on surgical practices and outcomes in orthopedic patients with metal implants. Arch Dermatol. 2012;148:687-93.

5. Hallab N, Merritt K, Jacobs JJ. Metal sensitivity in patients with orthopaedic implants. J Bone Jt Surg Am. 2001;83-A:428-36.
6. Mitchelson AJ, Wilson CJ, Mihalko WM, et al. Biomaterial hypersensitivity: is it real? Supportive evidence and approach considerations for metal allergic patients following total knee arthroplasty. Biomed Res Int. 2015;2015:137287.

7. Schalock PC, Thyssen JP. Metal hypersensitivity reactions to implants: opinions and practices of patch testing dermatologists. Dermat Contact Atopic Occup Drug. 2013;24:313-20.

8. Swiontkowski MF, Agel J, Schwappach J, McNair P, Welch M. Cutaneous metal sensitivity in patients with orthopaedic injuries. J Orthop Trauma. 2001;15:86-9

9. Davis MDP, Mowad CM, Scheinman P. Orthopedic prostheses: is there any point in patch testing? Dermat Contact Atopic Occup Drug. 2004;15:210-2.

10. Thyssen JP, Menné T, Schalock PC, Taylor JS, Maibach HI. Pragmatic approach to the clinical work-up of patients with putative allergic disease to metallic orthopaedic implants before and after surgery. Br J Dermatol. 2011;164:473-8.

11. Aquino M, Mucci T. Systemic contact dermatitis and allergy to biomedical devices. Curr Allergy Asthma Rep. 2013;13:518-27.

12. Granchi D, Cenni E, Giunti A, Baldini N. Metal hypersensitivity testing in patients undergoing joint replacement: a systematic review. J Bone Jt Surg Br. 2012;94:1126-34.

13. Bravo D, Wagner ER, Larson DR, Davis MP, Pagnano MW, Sierra RJ. No increased risk of knee arthroplasty failure in patients with positive skin patch testing for metal hypersensitivity: a matched cohort study. J Arthroplasty. 2016;31:1717-21.

14. Vos D, Hanson B, Verhofstad M. Implant removal of osteosynthesis: the Dutch practice. Results of a survey. J Trauma Manag Outcomes. 2012;6:6.

15. Fary C, Thomas GER, Taylor A, Beard D, Carr A, Glyn-Jones S. Diagnosing and investigating adverse reactions in metal on metal hip implants. BMJ. 2011;343:d7441.

16. Vermes C, Kuzsner J, Bárdos T, Than P. Prospective analysis of human leukocyte functional tests reveals metal sensitivity in patients with hip implant. J Orthop Surg Res. 2013;8:12.

17. Willert H-G, Buchhorn GH, Fayyazi A, et al. Metal-on-metal bearings and hypersensitivity in patients with artificial hip joints. A clinical and histomorphological study. J Bone Jt Surg Am. 2005;87:28-36.

18. Schalock PC, Menné T, Johansen JD, et al. Hypersensitivity reactions to metallic implants- 
diagnostic algorithm and suggested patch test series for clinical use. Contact Dermatitis. 2012;66:4-19.

19. Bircher A, Friederich NF, Seelig W, Scherer K. Allergic complications from orthopaedic joint implants: the role of delayed hypersensitivity to benzoyl peroxide in bone cement. Contact Dermatitis. 2012;66:20-6.

20. Maldonado-Naranjo AL, Healy AT, Kalfas IH. Polyetheretherketone (PEEK) intervertebral cage as a cause of chronic systemic allergy: a case report. Spine J. 2015;15:e1-3.

21. Kubba R, Taylor JS, Marks KE. Cutaneous complications of orthopedic implants. A two-year prospective study. Arch Dermatol. 1981;117:554-60.

22. Krecisz B, Kieć-Swierczyńska M, Bakowicz-Mitura K. Allergy to metals as a cause of orthopedic implant failure. Int $\mathrm{J}$ Occup Med Environ Health. 2006;19:178-80.

23. Stejskal V. Metals as a common trigger of inflammation resulting in non-specific symptoms: diagnosis and treatment. Isr Med Assoc J. 2014;16:753-8.

24. Granchi D, Cenni E, Tigani D, Trisolino G, Baldini $\mathrm{N}$, Giunti A. Sensitivity to implant materials in patients with total knee arthroplasties. Biomaterials. 2008;29:1494-500.

25. Rooker GD, Wilkinson JD. Metal sensitivity in patients undergoing hip replacement. A prospective study. J Bone Jt Surg Br. 1980;62-B:502-5.

26. Carlsson A, Möller H. Implantation of orthopaedic devices in patients with metal allergy. Acta Derm Venereol. 1989;69:62-6.

27. Gawkrodger DJ. Metal sensitivities and orthopaedic implants revisited: the potential for metal allergy with the new metal-on-metal joint prostheses. Br J Dermatol. 2003;148:1089-93.

28. Galbraith JG, Butler JS, Browne T-J, Mulcahy D, Harty JA. Infection or metal hypersensitivity? The diagnostic challenge of failure in metal-on-metal bearings. Acta Orthop Belg. 2011;77:145-51.

29. Granchi D, Cenni E, Trisolino G, Giunti A, Baldini N. Sensitivity to implant materials in patients undergoing total hip replacement. J Biomed Mater Res B Appl Biomater. 2006;77:257-64.

30. Thomas P, Geier J, Dickel H, et al. DKG statement on the use of metal alloy discs for patch testing in suspected intolerance to metal implants. J Dtsch Dermatol Ges. 2015;13:1001-4.
31. Schalock PC, Thyssen JP. Patch testers' opinions regarding diagnostic criteria for metal hypersensitivity reactions to metallic implants. Dermat Contact Atopic Occup Drug [Internet]. 2013 [cited 2016 Mar 14];24:183-5. http://www.ncbi.nlm.nih.gov/ pubmed/23857019.

32. Müller K, Valentine-Thon E, Müller KE. Hypersensitivity to titanium: clinical and laboratory evidence. Neuro Endocrinol Lett. 2006;27:31-3531.

33. Schalock PC, Crawford G, Nedorost S, et al. Patch testing for evaluation of hypersensitivity to implanted metal devices. Dermatitis. 2016;27:241-7.

34. Rostoker G, Robin J, Binet O, et al. Dermatitis due to orthopaedic implants. A review of the literature and report of three cases. J Bone Jt Surg Am. 1987;69:1408-12.

35. Razak A, Ebinesan AD, Charalambous CP. Metal allergy screening prior to joint arthroplasty and its influence on implant choice: a Delphi consensus study amongst orthopaedic arthroplasty surgeons. Knee Surg Relat Res. 2013;25:186-93.

36. Frigerio E, Pigatto PD, Guzzi G, Altomare G. Metal sensitivity in patients with orthopaedic implants: a prospective study. Contact Dermatitis. 2011;64:273-9.

37. Bravo D, Wagner ER, Larson DR, Davis MP, Pagnano MW, Sierra RJ. No increased risk of knee arthroplasty failure in patients with positive skin patch testing for metal hypersensitivity: a matched cohort study. J Arthroplasty. 2016;31:1717-21.

38. So S, Harris IA, Naylor JM, Adie S, Mittal R. Correlation between metal allergy and treatment outcomes after ankle fracture fixation. J Orthop Surg (Hong Kong). 2011;19:309-13.

39. Mowad CM. Practice gap: the role of patch testing in the selection and management of metal device implants. Arch Dermatol.. 2012;148:693-4.

40. Gawkrodger DJ. Nickel sensitivity and the implantation of orthopaedic prostheses. Contact Dermatitis. 1993;28:257-9.

41. Gupta R, Phan D, Schwarzkopf R. Total knee arthroplasty failure induced by metal hypersensitivity. Am J Case Rep. 2015;16:542-7.

42. Pacheco KA. Allergy to surgical implants. J Allergy Clin Immunol Pract. 2015;3:683-95.

43. Basko-Plluska JL, Thyssen JP, Schalock PC. Cutaneous and systemic hypersensitivity reactions to metallic implants. Dermat Contact Atopic Occup Drug. 2011;22:65-79. 
44. Morwood MP, Garrigues GE. Shoulder arthroplasty in the patient with metal hypersensitivity. J Shoulder Elbow Surg. 2015;24:1156-64.

45. Lützner J, Hartmann A, Dinnebier G, Spornraft-Ragaller P, Hamann C, Kirschner S. Metal hypersensitivity and metal ion levels in patients with coated or uncoated total knee arthroplasty: a randomised controlled study. Int Orthop. 2013;37:1925-31.

46. Grammatopoulos G, Pandit H, Kwon Y-M, et al. Hip resurfacings revised for inflammatory pseudotumour have a poor outcome. J Bone Jt Surg. 2009;9191:1019-24.

47. Bolognesi MP, Ledford CK. Metal-on-metal total hip arthroplasty. J Am Acad Orthop Surg. 2015;23:724-31.

48. Fabi D, Levine B, Paprosky W, et al. Metal-on-metal total hip arthroplasty: causes and high incidence of early failure. Orthopedics. 2012;35:e1009-16. 